\title{
NOUVELLE COCCIDIE DU FAISAN
}

\section{Par W.-L. YAKIMOFF et S.-N. MATSCHOULSKY}

Nous remercions le médecin-vétérinaire N. I. Agrinsky (Taschkent) de nous avoir envoyé les excréments de deux faisans (Phasianus chrysomelas).

On découvrit chez un des faisans un petit nombre d'oocystes de coccidies. Forme régulière, ovale ou légèrement allongée-ovale, avec enveloppe à double contour, d'épaisseur égale dans tout

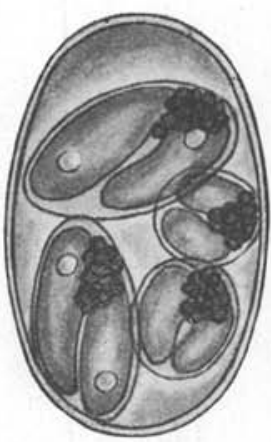

FIG. - Eimeria langeroni n. sp., du faisan (Phasianus chrysomelas!.

l'oocyste (jusqu'à $1,0 \mu$ ). Point de micropyle. Couleur de l'oocyste rose-jaunâtre. Dimensions : $30,0-36,0 \mu \times 16,0-20,0 \mu$, en moyenne $32,5 \mu \times 18,4 \mu$; les plus grands $36,0 \mu \times 20,0 \mu$, les plus petits $30,0 \mu \times 20,0 \mu$. Formindex $1: 0,47-0,67$, en moyenne $1: 0,57$.

Les oocystes développés avaient quatre spores; dimensions : $6,0-14,0 \mu \times 6,0 \mu$, sans corps de Schneider. Chaque sporocyste avait deux sporozoïtes, extrêmement massifs, et un reliquat. L'oocyste n'avait ni reliquat, ni granule polaire.

Mac Fadeyan, en Angleterre, et Sjöbring, en Suède, observèrent des coccidies de faisans, qu'on décrivit longtemps comme Eimeria tenella des poules. Tyzzer (1929) chez Phasianus colchicus torquatus, décrivit Eimeria phasiani. La forme est oblongue-elliptique.

Annales de Parasitologie, T. XV, $\mathrm{n}^{\circ} 2$. $-1^{\text {er }}$ mars 1937, p. 162-163. 
Après avoir sporulé, l'oocyste garde un granule polaire à l'un des pôles. Dimensions des oocystes : 19,8-26,4 $\mu \times 13,2-17,8 \mu$, en moyenne $23,04 \mu \times 15,89 \mu$. La sporulation se produit dans une solution de bichromate de potasse, à une température de $13-15^{\circ} \mathrm{C}$ pendant 24 heures. L'individualité de cette coccidie est prouvée par le fait que, alors que l'infection par Eimeria phasiani a été positive chez six faisans, elle a donné des résultats négatifs avec huit poulets, trois dindons et quatre cailles.

Nous remarquons que notre coccidie ne ressemble guère à l'Eimeria phasiani et qu'elle représente une nouvelle espèce, à laquelle nous donnons le nom Eimeria langeroni n. sp., en l'honneur du savant français M. Langeron.

Laboratoire des recherches scientifiques du Commissariat de l'Agriculture de l'U.R.S.S., pour les maladies à protozoaires des animaux domestiques (Directeur: Professeur W.-L. Yakimoff). 